Chapman University

Chapman University Digital Commons

Pharmacy Faculty Articles and Research

School of Pharmacy

$12-2004$

\title{
The Characterization of Nanosized Nickel-Zinc Ferrites Synthesized within Reverse Micelles of CTAB/1-Hexanol/Water Microemulsion
}

Vuk Uskoković

Chapman University, uskokovi@chapman.edu

Miha Drofenik

Jožef Štefan Institute

Irena Ban

Jožef Štefan Institute

Follow this and additional works at: http://digitalcommons.chapman.edu/pharmacy_articles

Part of the Other Chemistry Commons, and the Physical Chemistry Commons

\section{Recommended Citation}

Uskoković, V., Drofenik, M., Ban, I., 2004. The characterization of nanosized nickel-zinc ferrites synthesized within reverse micelles of CTAB/1-hexanol/water microemulsion. Journal of Magnetism and Magnetic Materials 284, 294-302. doi:10.1016/

j.jmmm.2004.06.051

This Article is brought to you for free and open access by the School of Pharmacy at Chapman University Digital Commons. It has been accepted for inclusion in Pharmacy Faculty Articles and Research by an authorized administrator of Chapman University Digital Commons. For more information, please contact laughtin@chapman.edu. 


\title{
The Characterization of Nanosized Nickel-Zinc Ferrites Synthesized within Reverse Micelles of CTAB/1-Hexanol/Water Microemulsion
}

\author{
Comments \\ NOTICE: this is the author's version of a work that was accepted for publication in Journal of Magnetism and \\ Magnetic Materials. Changes resulting from the publishing process, such as peer review, editing, corrections, \\ structural formatting, and other quality control mechanisms may not be reflected in this document. Changes \\ may have been made to this work since it was submitted for publication. A definitive version was subsequently \\ published in Journal of Magnetism and Magnetic Materials, volume 284, in 2006. DOI: 10.1016/ \\ j.jmmm.2004.06.051
}

The Creative Commons license below applies only to this version of the article.

\section{Creative Commons License}

\section{(C) $\oplus \Theta \Theta$}

This work is licensed under a Creative Commons Attribution-Noncommercial-No Derivative Works 4.0 License.

\section{Copyright}

Elsevier 


\title{
The Characterization of Nanosized Nickel-Zinc Ferrites Synthesized within Reverse Micelles of CTAB/1-Hexanol/Water Microemulsion
}

\author{
Vuk Uskoković, Miha Drofenik", Irena Ban* \\ »Jožef Stefan « Institute, Jamova 38, 1000 Ljubljana, Slovenia \\ *Faculty of Chemistry and Chemical Engineering, Smetanova 17, 2000 Maribor, Slovenia
}

\begin{abstract}
Stoichiometric nanocrystalline nickel-zinc ferrites were synthesized by a reverse micelle method following a multi-microemulsion approach. Different $\mathrm{pH}$ values were chosen for the alkali precipitating reaction during the synthesis of different powders. Synthesized, as-dried and subsequently calcined powders were characterized in terms of their magnetic properties. XRD analyses and specific-surface area measurements were used to determine the average particle sizes of the synthesized samples. DCS and TGA measurements were performed to reveal the phase transitions within the samples at elevated temperatures, whereas TEM was used to view and record the microstructure of the nanosized ferrite samples. A possible mechanism of the formation of the synthesized NiZn-ferrite was also discussed.
\end{abstract}

\section{Introduction}

Reverse micelles, which are essentially nanosized aqueous droplets that exist in microemulsions with certain compositions, are known to represent an excellent medium for the synthesis of nanoparticles with uniform morphologies ${ }^{1-3}$. By varying different parameters within a given microemulsion system, such as the concentrations or the molar ratios of constitutive components, it is said to be possible to design the structure, the properties and therefore the application of a synthesized product.

Nickel-zinc ferrites are magnetic materials that are much used by the modern electronics industry. This is primarily due to their high electrical resistivities, which implies low eddy-current losses that become significant at higher frequencies of electromagnetic fields. Nickel-zinc ferrites also exhibit high values of saturation magnetization, magnetic permeability, Curie temperature and dielectric constant, together with high mechanical strength, good chemical stability, low coercivities and low dielectric losses. The study presented here focused on the properties of nanocrystalline $\mathrm{Ni}_{0,5} \mathrm{Zn}_{0,5} \mathrm{Fe}_{2} \mathrm{O}_{4}$ synthesized within reverse micelles of CTAB/1-hexanol/water microemulsion.

\section{Experimental}

\subsection{Chemical Reagents}

The following chemicals were used in the synthesizing procedure: CTAB ( $>99 \%$, Alfa Aesar) as the surfactant; 1-hexanol ( $>98 \%$, Merck-Schuchardt) as the oil phase; deionized water as the aqueous phase of the microemulsion; $\mathrm{FeSO}_{4} \times 7 \mathrm{H}_{2} \mathrm{O}(>99 \%$, Alfa 
Aesar), $\mathrm{NiSO}_{4} \times 7 \mathrm{H}_{2} \mathrm{O}(99.7 \%$, Podnart $)$ and $\mathrm{ZnSO}_{4} \times 7 \mathrm{H}_{2} \mathrm{O}(>99 \%$, Alfa Aesar) as the precursor salts; $\mathrm{H}_{2} \mathrm{SO}_{4}$ (99.8 \%, Carlo Erba) as the acidifying agent; tetramethylammonium hydroxide $\left(\left(\mathrm{CH}_{3}\right)_{4} \mathrm{NOH}\right)(25 \mathrm{wt} \%$, 99.5\% purity, Alfa Aesar) as the precipitating agent; $\mathrm{H}_{2} \mathrm{O}_{2}$ (30 wt\%, Carlo Erba) as an oxidizing agent; and absolute ethanol (99.8\%, Carlo Erba) as the washing agent.

\subsection{Synthesis route}

The synthesizing procedure followed the multi-microemulsion approach. Three reverse micellar $\mathrm{CTAB} / 1$-hexanol/water microemulsions with identical weight ratios of their three basic constitutive components (CTAB : 1-hexanol $:$ water $=30.5: 57: 12.5)$ were prepared; one comprising an acidic $(\mathrm{pH}=1.6)$ stoichiometric aqueous $0.237-\mathrm{M}$ solution of sulphate salts of divalent precursor cations, the second comprising a $0.5-\mathrm{M}$ solution of $\left(\mathrm{CH}_{3}\right)_{4} \mathrm{NOH}$, which served as the precipitating agent; and the third comprising $30 \mathrm{wt} \%$ aqueous solution of $\mathrm{H}_{2} \mathrm{O}_{2}$. The first two microemulsions were mixed and stirred for one hour in air at room temperature. In the midst of an 1 hour aging time, the third microemulsion was added. The colloidal precipitate that formed was then sedimented by centrifuging and washing with ethanol and water.

\subsection{Characterization}

The as-dried and subsequently calcined powders were analyzed by using TEM (JEOL JEM-2000FX), DSC and TGA measurements (Mettler-Toledo STAR System), specificsurface area measurements (Micromeritics Gemini II), magnetic measurements (Manics DSM10 magnetometer) and X-ray diffraction analysis (D4 Endeavor diffractometer). Average particle sizes were estimated by using Debye-Scherrer's equation on the (311) diffraction peak of the spinel products, and specific-surface area measurements yielded another estimation of average particle sizes based on an assumption of spherical particles. The samples for TEM analysis were dispersed in ethanol with the aid of ultrasound, and then applied to a copper grid, where they were allowed to dry and later viewed on the TEM. DSC and TGA measurements were performed in air up to $500{ }^{\circ} \mathrm{C}$, with heating and cooling rates of $10{ }^{\circ} \mathrm{C} / \mathrm{min}$.

\section{Results and discussion}

The amount of precipitating agent used was shown to play a crucial role in the process of synthesizing the nanosized NiZn-ferrite particles. If the amount of precipitating agent was less than the stoichiometric amount needed to precipitate the precursor cations in the form of divalent hydroxides, the resulting powder was primarily made up of goethite $(\alpha-\mathrm{FeOOH})$. In other words, if the $\mathrm{pH}$ value of the precipitation was below 8 , goethite was formed, whereas if the $\mathrm{pH}$ of the precipitation larger was higher than 8, a spinel phase with better crystallinity was obtained (Fig. 1). Debye-Scherrer's equation was used to evaluate the average particle sizes, and the powders synthesized between precipitating $\mathrm{pH}$ values of 8 and 10 were found to have average particle sizes at the order of 2-3 nm, whereas powders synthesized at $\mathrm{pH}$ values higher than 10 had average particle sizes of $\sim 4 \mathrm{~nm}$ (Table 1). This size of the produced particles is 
consistent with the estimated diameters of the reverse micelles' »water pools « in herein used microemulsion system for the given water content ${ }^{4}$, used in these experiments.

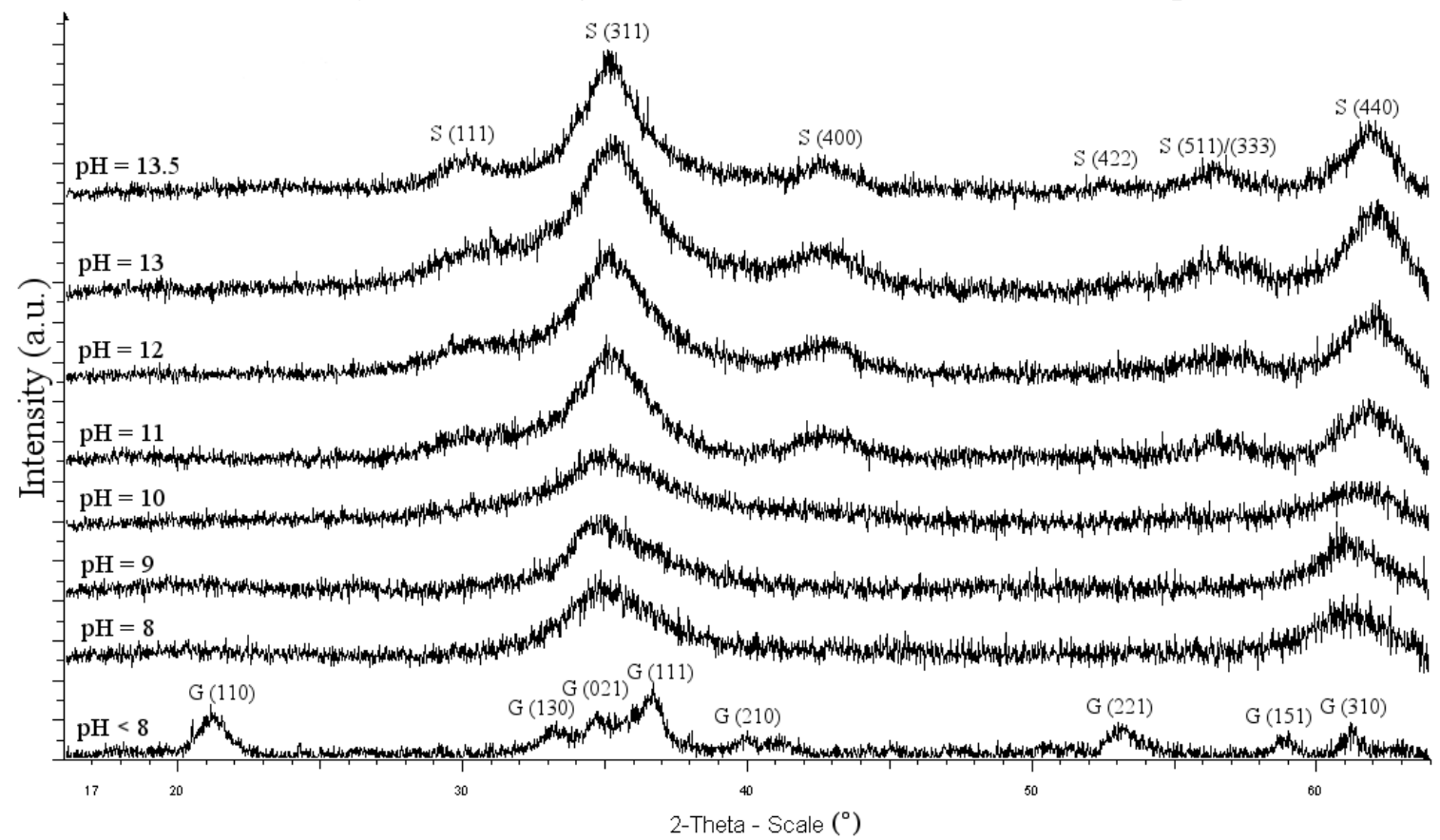

Fig.1. X-ray diffractograms of NiZn-ferrite powders synthesized within reverse micelles at different precipitating $\mathrm{pH}$ values. The diffraction peaks denoted with $\mathrm{S}$ are spinel-derived, whereas $\mathrm{G}$ denotes goethite-derived peaks.

Table 1

Average particle sizes of powders synthesized at different precipitating $\mathrm{pH}$ values, as deduced from XRD $\left(\mathrm{r}_{\mathrm{x}}\right)$ and specific-surface area $\left(\mathrm{r}_{\mathrm{s}}\right)$ measurements, and the room-temperature magnetization measured at the magnetic field of $12 \mathrm{kOe}\left(\mathrm{M}_{\mathrm{s}}\right)$.

\begin{tabular}{cccc}
\hline $\mathrm{pH}$ & $\mathrm{r}_{\mathrm{x}}(\mathrm{nm})$ & $\mathrm{r}_{\mathrm{s}}(\mathrm{nm})$ & $\mathrm{M}_{\mathrm{s}}(\mathrm{emu} / \mathrm{g})$ \\
\hline 8 & 2 & 6 & 1.2 \\
9 & 3 & 4 & 0.9 \\
10 & 2 & 4 & 1.1 \\
11 & 4 & 4 & 4.2 \\
12 & 4 & 5 & 4.6 \\
13 & 4 & 4 & 4.6 \\
13.5 & 4 & 4 & 5.4 \\
\hline
\end{tabular}

The average particle sizes deduced from the specific-surface area measurements were slightly higher than the values of the same quantity derived from the X-ray-diffraction lines-broadening analysis. This is probably a result of the agglomeration of nanosized particles, which occurs not during the chemical synthesizing procedure, but during the isolation of the particles from their parent microemulsion. The low values of magnetization at almost saturating field are consistent with the average particle sizes of the synthesized powders. This is because for particles of $3 \mathrm{~nm}$ in size, about half of all the constituent atoms are positioned on the surface of the particles, where magnetic interactions are known to be weaker than in the cores of more crystalline particles. 


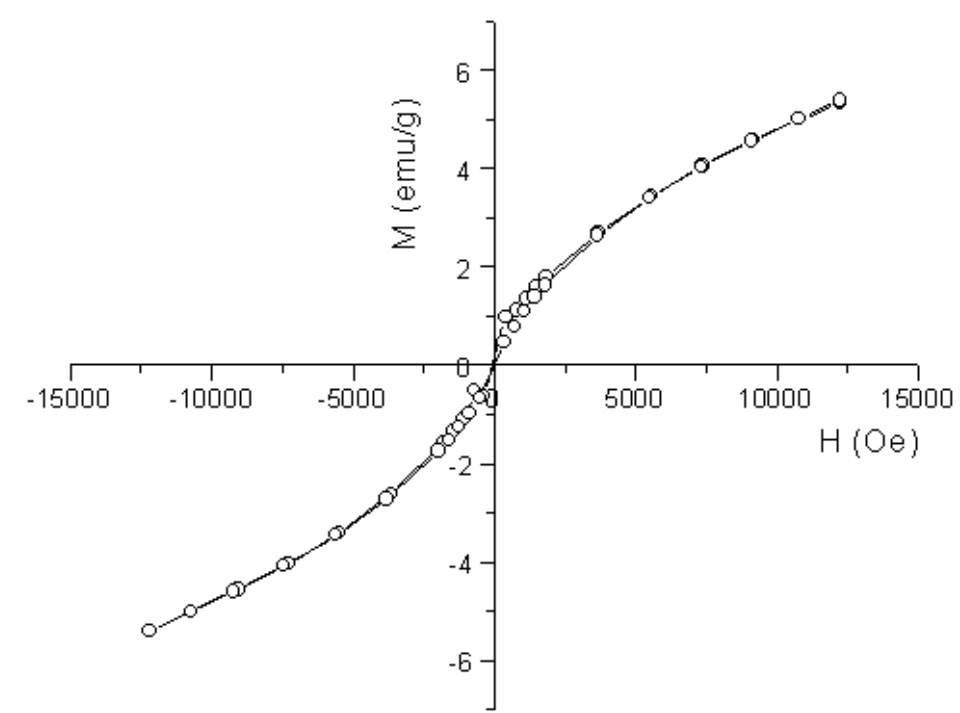

Fig. 2. Typical dependence of magnetization on the magnetic field for the as-dried powder

The hysteresis loop of the sample synthesized at $\mathrm{pH}=13.5$ (the shape of which is typical for the other samples as well) is shown in Fig. 2. The measured magnetization of the as-dried powders at the maximum magnetic field of $12 \mathrm{kOe}$ was found to vary with the $\mathrm{pH}$ value of the precipitation reaction (Fig.3): the higher the $\mathrm{pH}$ of the precipitation, the higher the magnetization of the powders. An increase in the measured magnetization between $\mathrm{pH}$ values of 10 and 11 can be correlated with the dependence of the average particle size on the $\mathrm{pH}$ of the precipitation (Table 1). The higher average particle sizes of the powders synthesized above $\mathrm{pH}$ values of 10 induce a higher magnetization, as a result of the improved crystallinity, which implies a better superexchange interaction. This increase in the average particle size with the increase in the precipitating $\mathrm{pH}$ value was already noticed for other types of wet approaches to the synthesis of ferrites ${ }^{5}$, and changes in the kinetic stability induced by increased $\mathrm{pH}$ values of the solution, which leads to the flocculation of smaller particles into larger aggregates, was attributed to this phenomenon $^{1}$. An increase in the average particle size has also been observed for the preparation of other non-ferrite, but oxide, materials ${ }^{6}$ within the same reverse micellar microemulsion system that was used in this work.

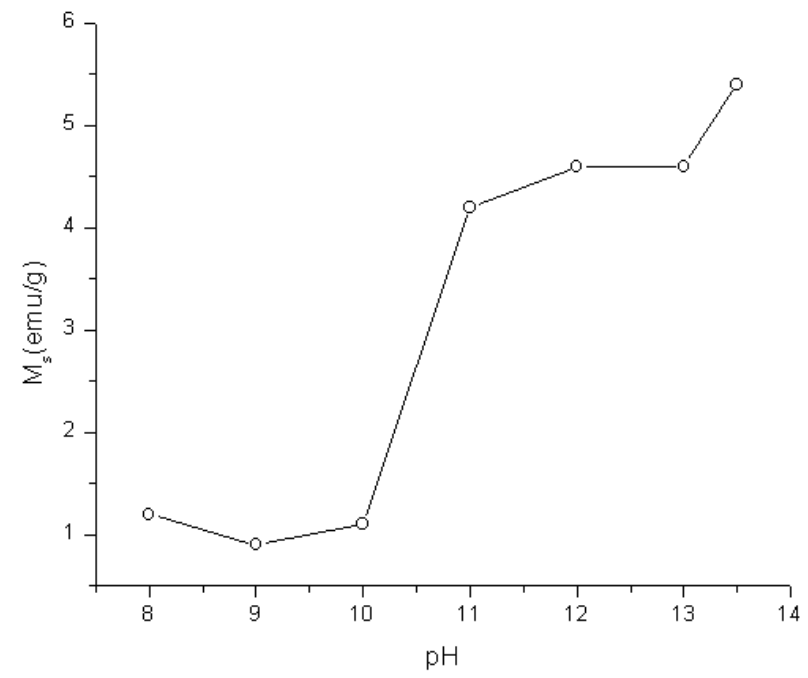


Fig 3. Magnetization of the synthesized samples measued at magnetic field of $12 \mathrm{kOe}$ versus precipitating $\mathrm{pH}$ value.

The NiZn-ferrites with particle sizes of less than approximately $20 \mathrm{~nm}$ are supposed to show superparamagnetic behaviour ${ }^{7}$. However, this is not the case with our synthesized samples, and we can ascribe this non-superparamagnetic behaviour to the agglomeration of nanosized particles, which is obvious from the TEM images.

Fig. 4 shows typical TEM micrographs of two synthesized powders: one at a precipitating $\mathrm{pH}$ value of 9 and the other at a $\mathrm{pH}$ value of 13 . Whereas in the first case, nanosized acicular particles are obvious within particle agglomerates, in the second, the higher precipitating $\mathrm{pH}$ value case, agglomerates comprising uniform spherical particles can be seen. The higher shape anisotropy of the particles synthesized at medium $\mathrm{pH}$ values (9-11) is reflected in very high coercivity values (1100 Oe) for a soft ferrite material. Since the spinel crystal structure, a characteristic of NiZn-ferrite, was detected in each synthesizing procedure of precipitating $\mathrm{pH} \geq 8$, it is possible that the lower $\mathrm{pH}$ values induce the formation of CTAB reverse micelles of worm-like shapes, whose existence was already described in the literature ${ }^{8,9,10}$, so that the acicular shape of the produced particles at lower $\mathrm{pH}$ values reflects the shape of the templating aqueous cores of the reverse micelles. It is already known that in the case of CTAB reversed or uninverted micelles, an alkali substance ${ }^{11}$, salts ${ }^{8}$ or co-surfactant ${ }^{12,13}$, might induce a spherical-to-worm-like transition, depending on the shape of the micelles. 1-hexanol is also known to play a role in CTAB microemulsions similar to that of the co-surfactant, because it is able to incorporate within an interface monolayer of surfactant molecules where it leads to the reduction of the repulsion of charged surfactant »heads « and induces the transition of spherical micelles into more elongated, elliptical ones ${ }^{14}$. The coexistence of spherical and worm-like micelles of CTAB molecules was also decribed in the literature ${ }^{13,15}$, and the fact that this phenomenon is possible might explain the coexistence of spherical and acicular ferrite particles in the case of medium precipitating $\mathrm{pH}$ value syntheses.

It is known that goethite, the dominant phase when the amount of precipitating agent used is less than the stoichiometric amount, often precipitates in the form of very long, acicular particles. Therefore, it is also possible that acicular particles within the samples synthesized at medium $\mathrm{pH}$ values might comprise ferri oxyhidroxide, which is known to be an intermediate compound ${ }^{16}$ within the formation of ferrite by a co-precipitation chemical procedure, which was used in this work as well. It was also noted ${ }^{17}$ that a strongly alkaline aqueous phase favors the formation of ferrite, whereas a low alkalinity favors the formation of $\alpha-\mathrm{FeOOH}$. Initial spherical $\mathrm{Fe}(\mathrm{OH})_{2}$ particles were observed to be gradually replaced by acicular $\alpha-\mathrm{FeOOH}$ particles ${ }^{18}$, so that the transition ratio was about 0.35 after $1 \mathrm{~h}$, and 0.96 after $6.5 \mathrm{~h}$. The transition ratio after $1 \mathrm{~h}$, which is equal to the aging time of NiZn-ferrite particles in our experiments, is approximately in accordance with the TEM images of the samples synthesized at the medium $\mathrm{pH}$ values (Fig. 3a). Thus, acicular particles present at the samples synthesized at medium $\mathrm{pH}$ values, might have amorphous poly-oxyhydroxide structure, which remain in the sample due to the long reaction times, and it is well-known that an increase in $\mathrm{pH}$ values leads to the increase in reaction times of the phase transformations ${ }^{19}$. Ferrihydrate $\left(\mathrm{Fe}_{2} \mathrm{O}_{3} \cdot \mathrm{xH}_{2} \mathrm{O}\right)$ is known to appear as the primary ferri-comprising intermediate compound in the formation 
of a ferrite only when the precipitating $\mathrm{pH}$ is below 9 . On the other hand, when $\mathrm{pH}>9, \delta$ $\mathrm{FeOOH} \rightarrow \alpha-\mathrm{FeOOH} \rightarrow \mathrm{Fe}_{3} \mathrm{O}_{4}$ is suggested mechanism of the formation of ferrous ferrite $^{20}$. Since the hexagonal form of $\mathrm{FeOOH}(\delta-\mathrm{FeOOH})$ has major diffraction peaks very close to the ones that are spinel-derived, within the samples of low crystallinity, considering high background noise, it is not possible to use ordinary X-ray diffraction analysis to make a qualitative distinction between these two phases. Therefore, we propose a possible model of chemical reactions that lead to the formation of ferrite by the precipitation of precursor constitutive-cation salts by alkali agent:

Stage I:

$$
\begin{aligned}
& \mathrm{Fe}^{2+}+1 / 4 \mathrm{O}_{2}+\mathrm{H}^{+} \rightarrow \mathrm{Fe}^{3+}+1 / 2 \mathrm{H}_{2} \mathrm{O} \\
& \mathrm{Fe}^{2+}+2 \mathrm{OH}^{-} \rightarrow \mathrm{Fe}(\mathrm{OH})_{2} \\
& \mathrm{Ni}^{2+}+\mathrm{Zn}^{2+}+4 \mathrm{OH}^{-} \rightarrow \mathrm{Ni}(\mathrm{OH})_{2}+\mathrm{Zn}(\mathrm{OH})_{2}
\end{aligned}
$$

Stage II:

$$
\begin{aligned}
& \qquad \mathrm{Fe}^{3+}+3 \mathrm{H}_{2} \mathrm{O}+\mathrm{H}^{+} \rightarrow \mathrm{Fe}(\mathrm{OH})_{3}+4 \mathrm{H}^{+} \\
& \text {sum of (1) and (4): } \mathrm{Fe}^{2+}+1 / 4 \mathrm{O}_{2}+5 / 2 \mathrm{H}_{2} \mathrm{O} \rightarrow \mathrm{Fe}(\mathrm{OH})_{3}+2 \mathrm{H}^{+} \\
& 4 \mathrm{Fe}(\mathrm{OH})_{2}+\mathrm{O}_{2} \rightarrow 4 \mathrm{FeOOH}+2 \mathrm{H}_{2} \mathrm{O}
\end{aligned}
$$

Stage IV:

$$
\mathrm{Fe}(\mathrm{OH})_{3} \rightarrow \mathrm{FeOOH}+\mathrm{H}_{2} \mathrm{O}
$$

$$
\text { Only if } \mathrm{pH}>8 \text {, }
$$$$
2 \mathrm{FeOOH}+1 / 2 \mathrm{Ni}(\mathrm{OH})_{2}+1 / 2 \mathrm{Zn}(\mathrm{OH})_{2} \rightarrow \mathrm{Ni}_{0,5} \mathrm{Zn}_{0,5} \mathrm{Fe}_{2} \mathrm{O}_{4}+2 \mathrm{H}_{2} \mathrm{O}
$$

where the reaction (7) is completed only if the $\mathrm{pH}>11$.

Oxygen necessary for the oxidation of precursor ferrous ions derives from the air dissolved in water used in the experiments, but on the other hand, oxidation of ferrous ions and subsequent formation of the ferrite based on decomposition of water molecules was also proved to be an energetically favorable process ${ }^{21}$. The mechanism of crystallization of ferrite particles usually assumes oxidation of ferrous into ferri ions, which then serve as crystallization nuclei ${ }^{22}$.

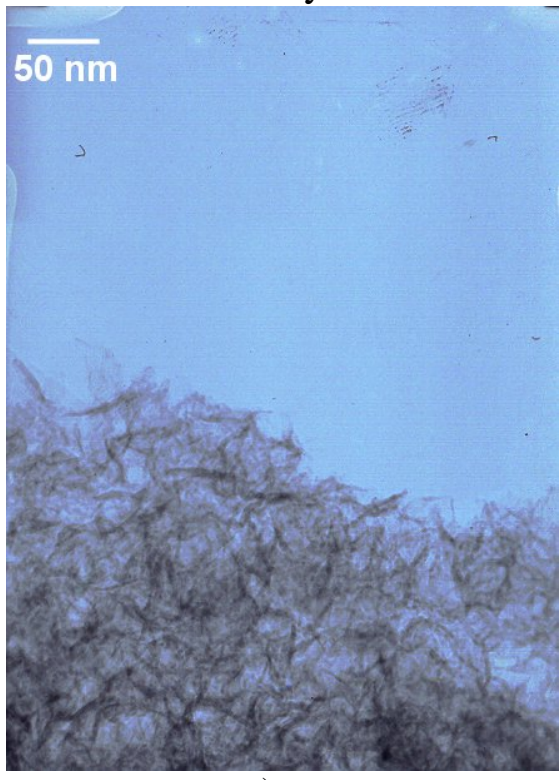

a.)

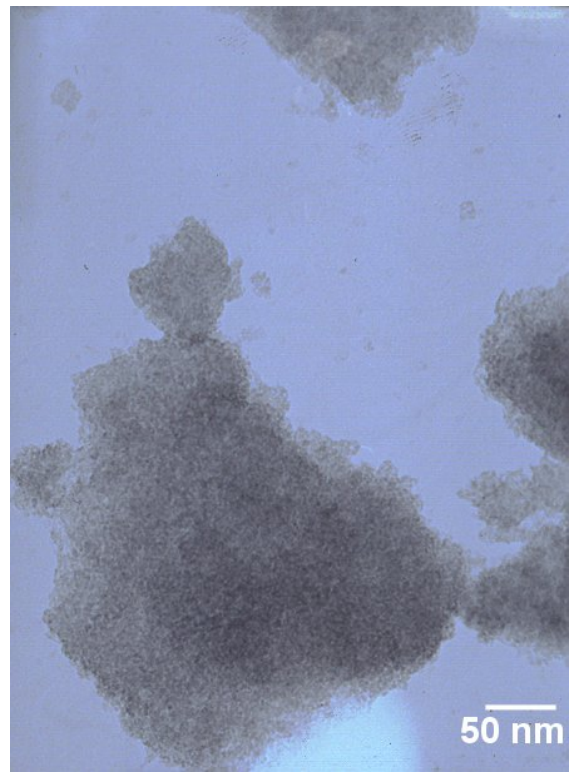

b.)

Fig.4a,b. TEM images of the samples synthesized at precipitating $\mathrm{pH}=9$ (a) and $\mathrm{pH}=13$ (b). 
Analytical measurements were performed in order to determine stoichiometry of the synthesized product. The stoichiometry obtained for the samples synthesized at the precipitating $\mathrm{pH}=9.7$ and 12 were $\mathrm{Ni}_{0.49} \mathrm{Zn}_{0.68} \mathrm{Fe}_{1.83} \mathrm{O}_{4}$ and $\mathrm{Ni}_{0.48} \mathrm{Zn}_{0.73} \mathrm{Fe}_{1.79} \mathrm{O}_{4}$, respectively, which suggest us that besides $\mathrm{XRD}$-diffraction-determined structure, there were no significant differences in elemental composition of the samples synthesized at medium and higher precipitating $\mathrm{pH}$ values as well, in spite of the difference in morphologies.

The as-dried powder synthesized at a precipitating $\mathrm{pH}=10$ was heated at different temperatures and the crystallinity of its spinel phase was found to improve with thermal treatment (Fig.5). Only the spinel phase is detected in all the heated powders as well as in all the as-dried powders. Increases in the average particle size, calculated from the XRD measurements, and the saturation magnetization with annealing temperature are shown in Fig.6a. Annealing at temperatures higher than $400{ }^{\circ} \mathrm{C}$ induces larger grain growth, and the increase in the average particle size is especially significant when the annealing is performed at $800{ }^{\circ} \mathrm{C}$. The growth of larger particles accompanied by the disappearance of smaller ones at temperatures higher than $400{ }^{\circ} \mathrm{C}$ is the main reason for the increase in the average particle size with the temperature of annealing, whereas the improved crystalline structure with a smaller S/V ratio resulting from thermal treatment leads to increasing values of saturation magnetization with annealing temperature. Both the saturation magnetization and the remanence were found to increase with increasing annealing temperatures up to $800{ }^{\circ} \mathrm{C}$ (Fig. 6b). The saturation magnetization of the sample annealed for 2 hours at $800{ }^{\circ} \mathrm{C}$ was $68 \mathrm{emu} / \mathrm{g}$, which is about $3 / 4$ of the saturation magnetization of stoichiometric NiZn-ferrites synthesized using a traditional high-temperature processing route.

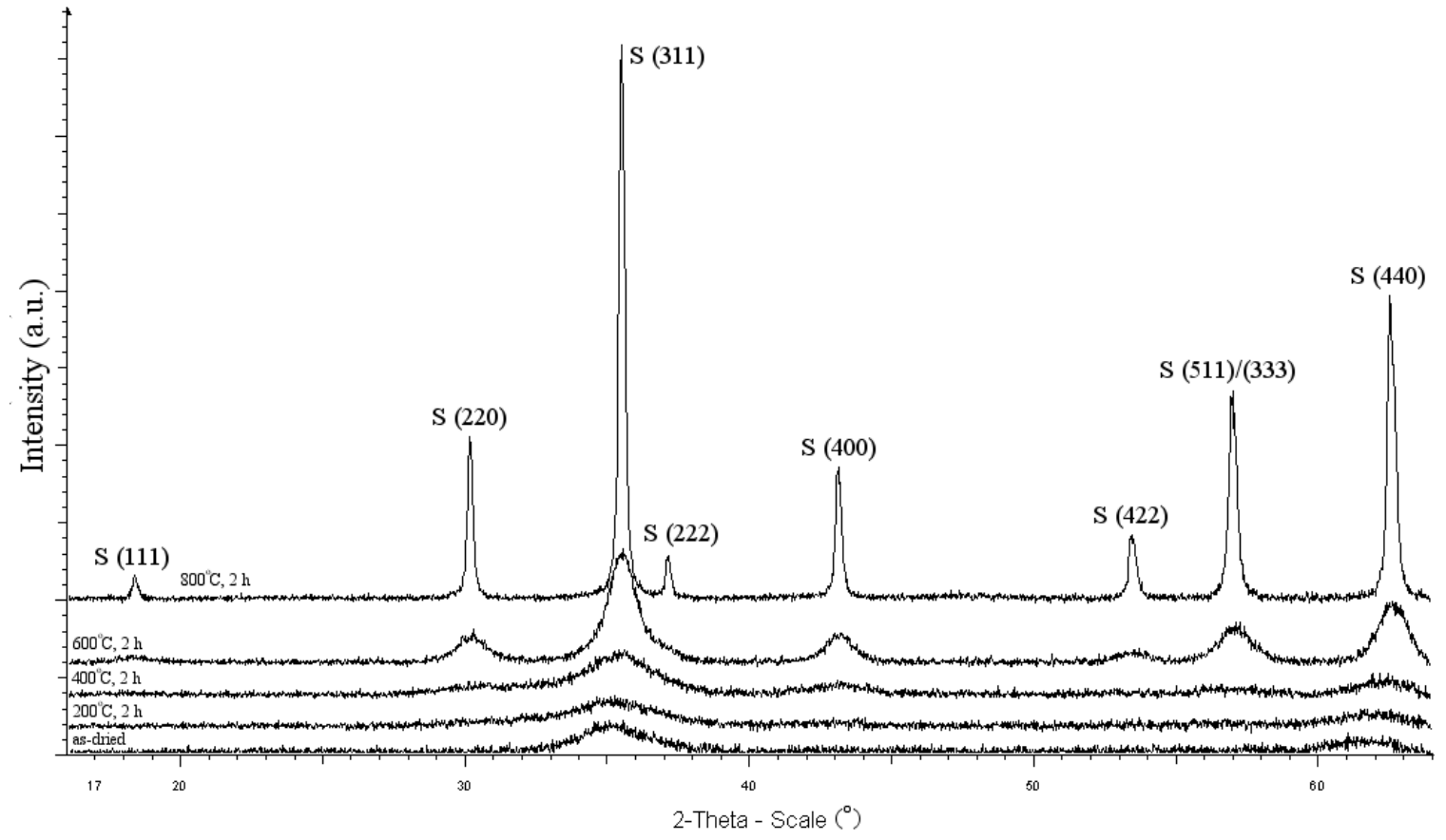

Fig. 5. X-ray diffractograms of the as-dried powder synthesized at precipitating $\mathrm{pH}=10$, and of the same powder annealed in air at temperatures of $200{ }^{\circ} \mathrm{C}, 400{ }^{\circ} \mathrm{C}, 600{ }^{\circ} \mathrm{C}$ and $800{ }^{\circ} \mathrm{C}$, in a bottom-up direction, for 2 hours each. $\mathrm{S}$ stands for spinel-phase-derived peaks. 


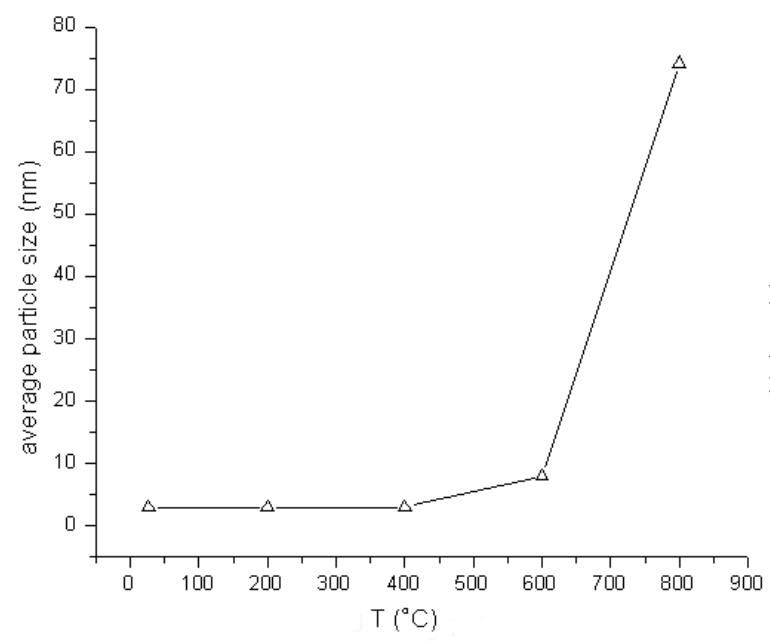

a.)

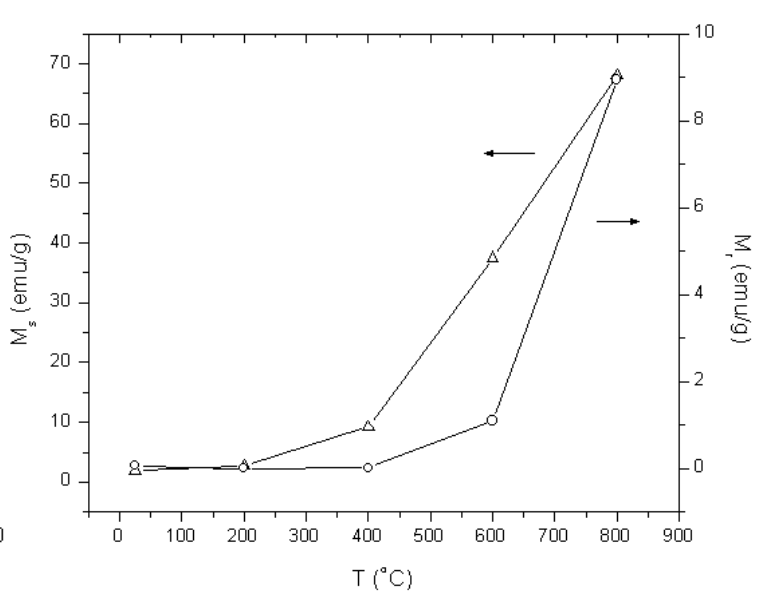

b.)

Fig. 6. Dependence of XRD-estimated average particle size (a), saturation magnetization (b, left Y-axis) and remanence (b, right $\mathrm{Y}$-axis) on the temperature of annealing.

TEM images of the sample annealed at $800^{\circ} \mathrm{C}$ are shown in Fig. 7. Coral-like agglomerates consist of particles, with sizes consistent with the average particle size determined from the X-ray diffraction-lines-broadening. There were no acicular particles present in the heated sample, which suggests us that the acicular particles were probably an intermediate phase during the formation of NiZn-ferrite.

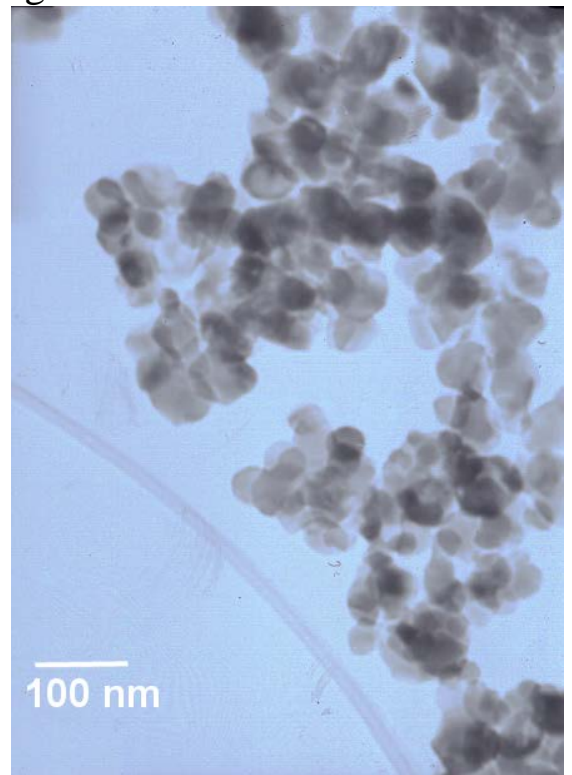

Fig. 7. TEM image of the NiZn-ferrite powder synthesized at the precipitating $\mathrm{pH}=10$ and annealed at 800 ${ }^{\circ} \mathrm{C}$ in air for $2 \mathrm{~h}$.

The heating thermogram with DSC diagrams in both heating and cooling regimes (10 ${ }^{\circ} \mathrm{C} / \mathrm{min}$ in air) of the sample synthesized at $\mathrm{pH}=13$ are shown in Fig.8. $25 \%$ of the sample evaporated while heating to $500{ }^{\circ} \mathrm{C}$, which is partly due to evaporated water and partly due to oxidised remains of hydrocarbon surfactant, whose molecules were, to a certain extent, attached to the surface of the ferrite particles, and could not be dettached even after multiple rinsing procedures. The largest weight loss occurs up to about $75{ }^{\circ} \mathrm{C}$, 
due to the evaporation of adsorbed water. If there were intermediate hydroxides present in the as-dried sample, a sharp weight-loss peak would have been evident at about 280 ${ }^{0} \mathrm{C}$, since hydroxides would be turned to oxides at about this temperature ${ }^{23}$. As this weight loss is missing in the recorded thermogram (Fig. 8a), the intermediate hydroxide of the spinel ferrite constitutive cations were turned into oxides, that is ferrite, in the microemulsion.

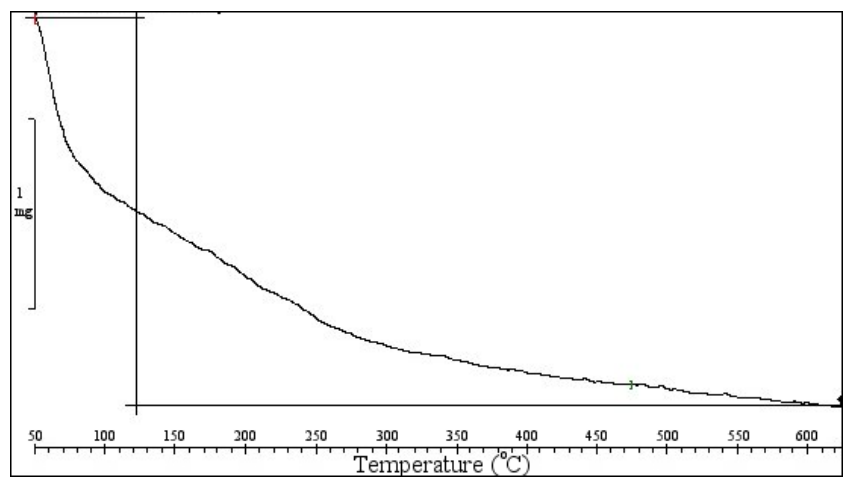

a.)

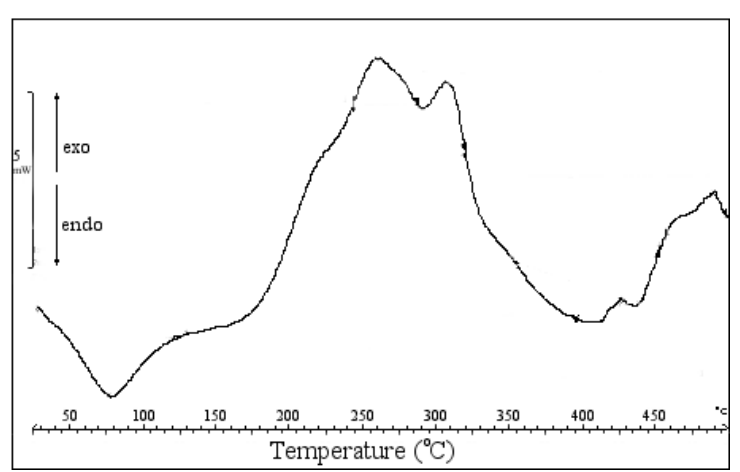

b.)

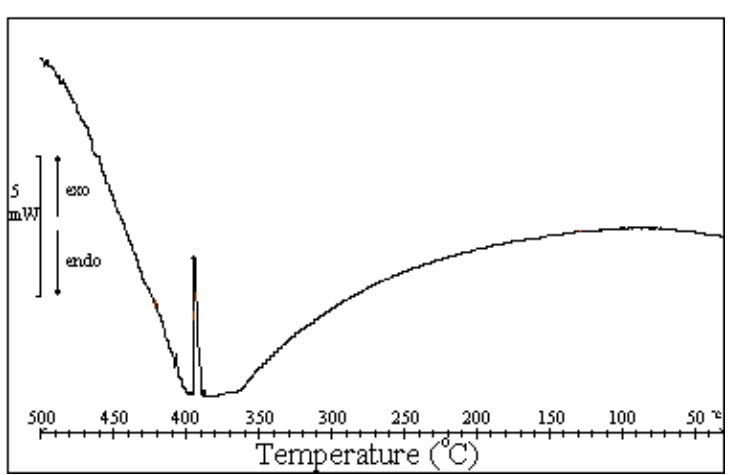

c.)

Fig. 8. Thermogram (a) and DSC diagrams in heating (b) and cooling regimes (c) of the sample synthesized at $\mathrm{pH}=13$.

The endothermic DSC peak at $75{ }^{\circ} \mathrm{C}$ is attributed to water and ethanol evaporation, two exothermic peaks, one at $260{ }^{\circ} \mathrm{C}$ and the other at $310{ }^{\circ} \mathrm{C}$, are separately attributed to an amorphous-to-crystalline phase transition and the oxidation of the remaining surfactant molecules. A thermal analysis of nanocrystalline ferrite powders is known to exhibit one or more exothermic peaks ${ }^{24}$ in the temperature range of $200-350{ }^{\circ} \mathrm{C}$, which is exactly the range where two exothermic peaks are visible in the heating regime. Both thermal processes are irreversibile, since no analogous changes were observed in the cooling regime. This could confirm the assumption that at least one of these exothermic peaks is derived from rearranging the spinel crystal structure by thermal activation ${ }^{25}$, that is from amorphous-to-crystalline phase transition ${ }^{26}$. Since $\mathrm{NiFe}_{2} \mathrm{O}_{4}$ and $\mathrm{Fe}_{3} \mathrm{O}_{4}$ both possess inverse spinel crystal structures, it was suggested that the transition between the inverted and regular spinel crystal structure should leave a trace on a DSC diagram ${ }^{27}$. However, it was shown that the inverted-to-regular spinel structure transition might occur only at temperatures higher than $300{ }^{\circ} \mathrm{C}$, since the time needed to equlibrate the distribution is very large $e^{28}\left(10^{4}\right.$ hours or more) at temperatures lower than $300{ }^{\circ} \mathrm{C}$. If either $\alpha$ - or $\gamma$ - forms of $\mathrm{FeOOH}$ (which are known to be needle-shaped) were present in 
the sample, a strong endothermic effect of the dehydration of $\mathrm{FeOOH}$ into $\mathrm{Fe}_{2} \mathrm{O}_{3}$ should have been present at the temperature range ${ }^{29}$ of $220-320{ }^{\circ} \mathrm{C}$.

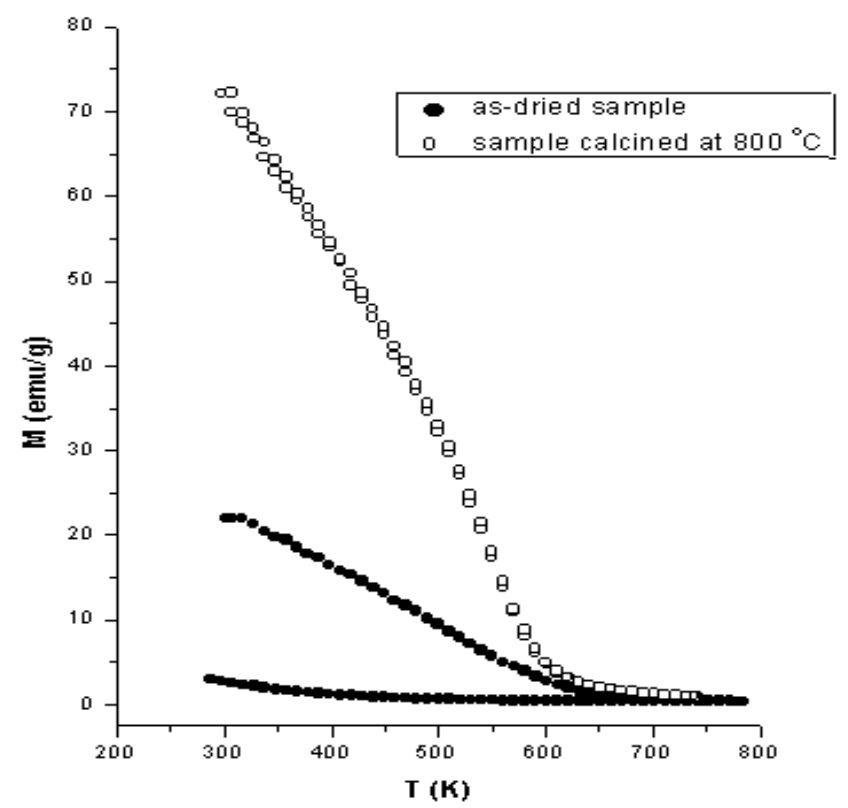

Fig. 9. Dependence of saturation magnetization versus temperature for the sample synthesized at $\mathrm{pH}=13$.

Bulk-synthesized NiZn-ferrites possess Curie transitions at around $490{ }^{\circ} \mathrm{C}$, but with decreasing particle size, it is known that the temperature of this phase transition decreases as well $^{30}$. Generally, a Curie transition in NiZn-ferrites, with no respect to average particle size is expected ${ }^{30}$ at temperatures of between $300{ }^{\circ} \mathrm{C}$ and $500{ }^{\circ} \mathrm{C}$. The endothermic peak that occurs within the heating regime at $396{ }^{\circ} \mathrm{C}$ has its corresponding exothermic peak in the cooling regime at $388{ }^{\circ} \mathrm{C}$, and both are attributed to the Curie transition. The temperature of $\sim 380{ }^{\circ} \mathrm{C}$ was obtained for Curie transition by following dependence of saturation magnetization of both as-dried and calcined samples versus temperature (Fig. 9). The exothermic Curie transition-derived peak during the cooling regime is much sharper in comparison with the respective endothermic peak within the heating regime. During the heat treatment, the nanosized grains are subjected to a coarsening processes, and thus during the cooling of the magnetically symmetrical and coarsened powder, the Curie transition is revealed as a sharper DSC peak. It is also possible that the Curie transition-derived endothermic DSC peak overlaps possibly present, in our case, only irreversible exothermic peaks. Enthalpy changes for the Curie transitions, derived from the calculated peaks' surface areas, have values of $-608.69 \mathrm{~mJ}$ for the endothermic transition and $806.24 \mathrm{~mJ}$ for the respective, exothermic phase transition. Improved crystallinity of the powder heated up to $500{ }^{\circ} \mathrm{C}$, and thus better restored superexchange interaction between neighbouring ions, might be the reason for the larger value of enthalpy change for the Curie transition in the cooling regime, comparing to the Curie-transition-derived enthalpy change in the heating regime.

\section{Conclusions}

The reverse micelle synthesis of nanocrystalline nickel-zinc ferrites can be successfully performed by following a multi-microemulsion approach at $\mathrm{pH}>8$ with an 
acidic solution of sulphate salts of precursor cations in one, and an aqueous 0.5-M solution of $\left(\mathrm{CH}_{3}\right)_{4} \mathrm{NOH}$ in the other, precipitating microemulsion. The average particle size was found to increase with the $\mathrm{pH}$ of the precipitation, with a particularly noticable increase between $\mathrm{pH}$ values of 10 and 11 . The magnetization measured at magnetic field of $12 \mathrm{kOe}$ was also found to increase with the increase in the precipitating $\mathrm{pH}$ values from 9 to 13.5, with a pronounced increase also between $\mathrm{pH}=10$ and $\mathrm{pH}=11$. The TEM images revealed the partly acicular nature of the synthesized particles at lower $\mathrm{pH}$ values, close to $\mathrm{pH}=10$. On the other hand, particles synthesized at higher $\mathrm{pH}$ values, close to 13, were spherical and of uniform size. This was explained by the existence of worm-like reverse micelles, together with the spherical micelles, so that the former gave rise to acicular particles, while the latter micelles induced the formation of spherical particles. The non-completion of the chemical route, which eventually leads to the formation of ferrite particles at higher $\mathrm{pH}$ values, might be taken into account to occur within the powder preparations performed at medium $\mathrm{pH}$ values. Thermal analysis and $\mathrm{M}_{\mathrm{s}}=\mathrm{f}(\mathrm{T})$ measurements revealed Curie transition at around $390{ }^{\circ} \mathrm{C}$ and two exothermic peaks at $260{ }^{\circ} \mathrm{C}$ and $310{ }^{\circ} \mathrm{C}$, which were attributed to an amorphous-to-crystalline phase transition. The crystallinity of the spinel samples was found to improve with the heat treatment, with the largest increase in XRD-determined average particle size for the samples annealed at $600{ }^{\circ} \mathrm{C}$ and $800{ }^{\circ} \mathrm{C}$. The saturation magnetization and remanence were also found to increase with annealing. TEM investigation of the sample annealed at $800{ }^{\circ} \mathrm{C}$ revealed a coral-like structure of larger and relatively uniform-sized rounded particles.

\section{References}

1. D. O. Yener, H. Giesche - "Synthesis of Pure and Manganese-, Nickel-, and ZincDoped Ferrite Particles in Water-in-Oil Microemulsions”, Journal of the American Ceramic Society 84 (9) 1987-95 (2001).

2. A. Nanni, L. Dei - “Ca(OH) 2 Nanoparticles from W/O Microemulsions”, Langmuir 19, 933 - 938 (2003).

3. L. M. Gan, L. H. Zhang, H. S. O. Chan, C. H. Chew, B. H. Loo - "A Novel Method for the Synthesis of Perovskite-type Mixed Metal Oxides by the Inverse Microemulsion Technique”, Journal of Materials Science 31, 1071 - 1079 (1996).

4. E. Rodenas, M. Valiente - "The Determination of Some Physical Properties of Reverse CTAB Micelles in 1-Hexanol”, Colloids and Surfaces 62, 289 - 295 (1992).

5. X. Jiao, D. Chen, Y. Hu - "Hydrothermal Synthesis of Nanocrystalline $\mathrm{M}_{\mathrm{x}} \mathrm{Zn}_{1-\mathrm{x}} \mathrm{Fe}_{2} \mathrm{O}_{4}$ $(\mathrm{M}=\mathrm{Ni}, \mathrm{Mn}, \mathrm{Co} ; \mathrm{x}=$ 0,40 - 0,60) Powders”, Materials Research Bulletin 37, 1583 1588 (2002).

6. L. Sun, Y. Zhang, J. Zhang, C. Yan, C. Liao, Y. Lu - "Fabrication of Size Controllable $\mathrm{YVO}_{4}$ Nanoparticles via Microemulsion-Mediated Synthetic Process", Solid State Communications 124, 35 - 38 (2002).

7. C. Caizer, M. Stefanescu - "Nanocrystallite Size Effects on $\sigma_{s}$ and $H_{c}$ in Nanoparticle Assemblies”, Physica B 327, 129 - 134 (2003).

8. Y. Liu, W. Wang, Y. Zhan, C. Zheng, G. Wang - "A Simple Route to Hydroxyapatite Nanofibers”, Materials Letters 56, 496 - 501 (2002).

9. S. Xu, H. Zhou, J. Xu, Y. Li - "Synthesis of Size-Tunable Silver Iodide Nanowires in Reverse Micelles”, Langmuir 18, 10503 - 10504 (2002). 
10. M. P. Pileni, A. Hammounda, I. Lisiecki, L. Motte, N. Moumen, J. Tanori - “Control of the Size and Shape of Nanoparticles”, in Fine Particles Science and Technology, edited by E. Pelizzetti, Kluwer Academic Publishers, 413 - 429 (1996).

11. F. G. Sanchez, C. C. Ruiz - "Intramicellar Energy Transfer in Aqueous CTAB Solutions”, Journal of Luminiscence 69, 179 - 186 (1996).

12. Z. Lin, J. J. Cai, L. E. Scriven, H. T. Davis - "Spherical-to-Wormlike Micelle Transition in CTAB Solutions”, Journal of Physical Chemistry 98, 5984 - 5993 (1994).

13. X. Fang, C. Yang - “An Experimental Study on the Relationship between the Physical Properties of CTAB/Hexanol/Water Reverse Micelles and $\mathrm{ZrO}_{2}-\mathrm{Y}_{2} \mathrm{O}_{3}$ Nanoparticles Prepared”, Journal of Colloid and Interface Science 212, 242 - 251 (1999).

14. B. Lindman, H. Wennerstrom - "Micelles. Amphiphile Aggregation in Aqueous Solution" in Topics in Current Chemistry 87: Micelles, Springer-Verlag Berlin Heidelberg, 1980.

15. J. Yang - "Viscoelastic Wormlike Micelles and their Applications", Current Opinion in Colloid \& Interface Science 7, 276 - 281 (2002).

16. G. Bate - "Recording Materials", in Ferromagnetic Materials, edited by E. P. Wohlfarth, North-Holland Publishing Company (1980).

17. Z. H. Zhou, J. Wang, X. Liu, H. S. O. Chan - "Synthesis of $\mathrm{Fe}_{3} \mathrm{O}_{4}$ Nanoparticles from Emulsions”, Journal of Materials Chemistry 11 (6), 1704 - 1709 (2001).

18. C. Li, S. Cai, T. Fang - "Rheological Behavior of Aciculate Ultrafine $\alpha-\mathrm{FeOOH}$ Particles under Alkaline Conditions”, Journal of Solid State Chemistry 141, 94 - 98 (1998).

19. C. Domingo, R. Rodriguez-Clemente - "The Pathways to Spinel Iron Oxides by Oxidation of Iron (II) in Basic Media”, Materials Research Bulletin 26, 47 - 55 (1991).

20. A. V. Tolchev, D. G. Kleschov, R. R. Bagautdinova, V. Yu. Pervushin “Temperature and $\mathrm{pH}$ Effect on Composition of a Precipitate Formed in $\mathrm{FeSO}_{4}-\mathrm{H}_{2} \mathrm{O}$ $\mathrm{H}^{+} / \mathrm{OH}^{-} \mathrm{H}_{2} \mathrm{O}_{2}$ System”, Materials Chemistry and Physics 74, 336 - 339 (2002).

21. Y. Todaka, M. Nakamura, S. Hattori, K. Tsuchiya, M. Umemoto - "Synthesis of Ferrite Nanoparticles by Mechanochemical Processing Using a Ball Mill”, Materials Transactions 44 (2) 277 - 284 (2003).

22. H. Robbins - "The Preparation of Mn-Zn Ferrites by Co-Precipitation”, in FERRITES: Proceedings of the International Conference, September - October 1980, Japan, Center for Academic Publications Japan (1981).

23. J. Fang, J. Wang, L. M. Gan, S. C. Ng, J. Ding, X. Liu - “Fine Strontium Ferrite Powders from an Ethanol-Based Microemulsion”, Journal of the American Ceramic Society 83 (5) 1049 - 1055 (2000).

24. W. C. Kim, S. J. Kim, Y. R. Uhm, C. S. Kim - "Magnetic Properties of NiZnCu Ferrite Powders and Thin Films Prepared by a Sol-Gel Method”, IEEE Transactions on Magnetics 37, 4 (2001).

25. J. Wang, P. F. Chong, S. C. Ng, L. M. Gan - "Microemulsion Processing of Manganese Zinc Ferrites”, Materials Letters 30, 217 - 221 (1997).

26. X. He, Q. Zhang, Z. Lang - "Kinetics and Magnetic Properties of Sol-Gel Derived NiZn Ferrite - $\mathrm{SiO}_{2}$ Composites”, Materials Letters 57, 3031 - 3036 (2003). 
27. N. S. Gajbhiye, G. Balaji - "Synthesis, Reactivity, and Cations Inversion Studies of Nanocrystalline $\mathrm{MnFe}_{2} \mathrm{O}_{4}$ Particles”, Thermochimica Acta 385 (2002) 143 - 151.

28. Z. Simsa, V. A. M. Brabers - "Influence of the Degree of Inversion on Magnetic Properties of $\mathrm{MnFe}_{2} \mathrm{O}_{4}$ ”, IEEE Transactions on Magnetics 11, 5 (1975).

29. I. Mitov, D. Paneva, B. Kunev - “Comparative Study of the Thermal Decomposition of Iron Oxyhydroxides”, Thermochimica Acta 386, 179 - 188 (2002).

30. K. V. P. M. Shafi, Y. Koltypin, A. Gedanken, R. Prozorov, J. Balogh, J. Lendvai, I. Felner - "Sonochemical Preparation of Nanosized Amorphous $\mathrm{NiFe}_{2} \mathrm{O}_{4}$ Particles", Journal of Physical Chemistry B 101, 6409 - 6414 (1997). 